PROCEEDINGS OF THE

AMERICAN MATHEMATICAL SOCIETY

Volume 130, Number 8, Pages 2183-2187

S 0002-9939(02)06595-4

Article electronically published on March 8, 2002

\title{
EQUIVARIANT RESOLUTION OF POINTS OF INDETERMINACY
}

\author{
Z. REICHSTEIN AND B. YOUSSIN
}

(Communicated by Michael Stillman)

\begin{abstract}
We prove an equivariant form of Hironaka's theorem on elimination of points of indeterminacy. Our argument uses canonical resolution of singularities and an extended version of Sumihiro's equivariant Chow lemma.
\end{abstract}

\section{INTRODUCTION}

Throughout this note we shall work over an algebraically closed field $k$ of characteristic 0. All algebraic varieties, schemes, groups, and all maps between them will be defined over $k$.

Let $G$ be a linear algebraic group (not necessarily connected). The main objects of interest for us will be algebraic varieties with a $G$-action; we will refer to them as $G$-varieties. A $G$-equivariant morphism between two such varieties will be called a morphism of $G$-varieties. The terms "rational map of $G$-varieties", "birational morphism of $G$-varieties", "birational isomorphism of $G$-varieties", etc., are defined in a similar manner.

Hironaka's theorem on elimination of points of indeterminacy (see $\mathrm{Hi}, \S 0.5$, Question E and Main Theorem II]) asserts that every rational map $f: X \rightarrow Y$ can be resolved into a regular map by a sequence of blowups $\pi: X_{m} \longrightarrow \ldots \longrightarrow X_{0}=X$ with smooth centers. In other words, $\pi$ can be chosen so that the composition $f \pi$ is regular. The purpose of this paper is to prove the following equivariant version of this result.

Theorem 1. Let $f: X \rightarrow Y$ be a rational map of $G$-varieties, where $Y$ is complete. Then there is a sequence of blowups

$$
\pi: X_{m} \longrightarrow X_{m-1} \longrightarrow \ldots \longrightarrow X_{1} \longrightarrow X_{0}=X
$$

with smooth $G$-invariant centers such that the composition $f \pi$ is regular.

Our proof will rely on canonical resolution of singularities. Along the way we prove an equivariant form of Chow's lemma (Proposition[2), generalizing a theorem of Sumihiro ([Su, Theorem 2]). For applications of Theorem 1, see [RY1, Remark 5.4] and [RY2] Remark 6.3].

Received by the editors September 29, 2000.

2000 Mathematics Subject Classification. Primary 14E15, 14L30.

Key words and phrases. Group action, blowing up, resolution of singularities, equivariant map. Z. Reichstein was partially supported by NSF grant DMS-9801675. 


\section{Equivariant ChOW LEMma}

In this section we will prove the following generalization of Chow's lemma.

Proposition 2. For every $G$-variety $X$, there exist a quasiprojective $G$-variety $Z$ and a proper birational morphism $Z \longrightarrow X$. If $X$ is complete, then $Z$ is projective.

Note that if $G$ is assumed to be connected, this result is a well-known theorem of Sumihiro [Su, Theorem 2]; see also [PV, Theorem 1.3]. The argument below reduces the general case to the case where $G$ is connected.

Proof. The second assertion is an immediate consequence of the first: if $X$ is complete, $Z \longrightarrow X$ is proper and $Z$ is quasiprojective, then $Z$ is also complete and, hence, projective.

To prove the first assertion, let $G_{0}$ be the connected component of $G$. Applying Sumihiro's theorem to $X$, viewed as a $G_{0}$-variety, yields a quasiprojective $G_{0}$-variety $Y$ and a proper $G_{0}$-equivariant birational morphism $f: Y \longrightarrow X$.

For the rest of this proof we shall use set-theoretic notation: by a "point" we will always mean a closed point.

Recall that the homogeneous fiber product $G *_{G_{0}} \quad Y$ is the $G$-variety defined as the geometric quotient $(G \times Y) / G_{0}$ for the action of $G_{0}$ given by $g_{0}(g, y)=$ $\left(g g_{0}^{-1}, g_{0} y\right)$, where $g \in G, g_{0} \in G_{0}$ and $y \in Y$; see $\mathrm{PV}$ Section 4.8]. We shall write $[g, y]$ for the element of $G *_{G_{0}} Y$ represented by $(g, y) \in G \times Y$. Since $G_{0}$ has finite index in $G, G *_{G_{0}} Y$ admits a more concrete description as a disjoint union of $\left|G / G_{0}\right|$ copies of $Y$. More precisely, if we choose a representative $a_{h}$ for each $h \in G / G_{0}$, we can explicitly identify $G / G_{0} \times Y$ and $G *_{G_{0}} Y$ as abstract varieties, via $(h, y) \mapsto\left[a_{h}, y\right]$. Moreover, if we define a $G$-action on $G / G_{0} \times Y$ by $g(h, y) \longrightarrow\left(\bar{g} h,\left(a_{h}^{-1} g^{-1} a_{\bar{g} h}\right) y\right)$, then $(h, y) \mapsto\left[a_{h}, y\right]$ identifies $G / G_{0} \times Y$ and $G *_{G_{0}} Y$ as $G$-varieties. Here $\bar{g}$ is the image of $g$ in $G / G_{0}$, and $\left(a_{h}^{-1} g^{-1} a_{\bar{g} h}\right) y$ is well-defined because $a_{h}^{-1} g^{-1} a_{\bar{g} h}$ is an element of $G_{0}$.

Let $\alpha: G *_{G_{0}} Y \longrightarrow X$ and $\beta: G *_{G_{0}} Y \longrightarrow G / G_{0}$ be the maps of $G$-varieties given by $\alpha:[g, y] \mapsto g f(y)$ and $\beta:[g, y] \mapsto \bar{g}$. (Here $G$ acts on $G / G_{0}$ by left multiplication.) These maps are shown in the diagram below:

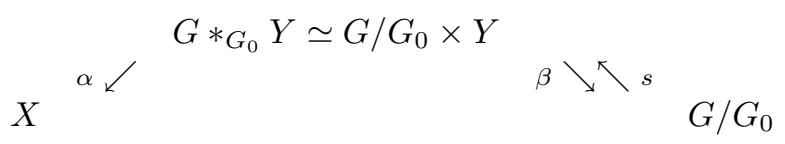

Let $S$ be the set of all sections $s$ of $\beta$. Note that if we identify $G *_{G_{0}} Y$ with $G / G_{0} \times Y$ as above, then $\beta: G / G_{0} \times Y \longrightarrow G / G_{0}$ is the projection to the first factor. Thus $S \simeq Y^{\left|G / G_{0}\right|}$ as an abstract variety. Moreover, since $\beta$ is $G$-equivariant, $G$ acts on this variety by $g: s \mapsto t$, where $s, t \in S$ and $t(h)=g \cdot s\left(\bar{g}^{-1} h\right)$ for any $h \in G / G_{0}$.

Let $Z$ be the closed $G$-invariant subvariety of $S$ consisting of those sections $s: G / G_{0} \longrightarrow G *_{G_{0}} Y \simeq G / G_{0} \times Y$ with the property that $\alpha \circ s\left(G / G_{0}\right)$ is a single point of $X$; we shall denote this point by $x_{s}$.

We claim that the morphism $\phi: Z \longrightarrow X$ given by $s \longrightarrow x_{s}$ has the properties asserted in the proposition. Indeed, since $Y$ is quasiprojective, and $Z$ is a closed subvariety of $S \simeq Y^{\left|G / G_{0}\right|}, Z$ is quasiprojective as well.

To show that $\phi$ is a birational morphism, assume the birational morphism $f: Y \longrightarrow X$ is an isomorphism over a dense open subset $U \subset X$. Then $V=$ 
$\bigcap_{h \in G / G_{0}} a_{h} U$ is also a dense open subset of $X$, and for every $x \in V, \alpha^{-1}(x)=$ $\left\{\left[a_{h}, f^{-1}\left(a_{h^{-1}} x\right)\right]: h \in G / G_{0}\right\}$; it is the image of the unique section $s_{x} \in S$ satisfying $x_{s_{x}}=x$. This section is given by $s_{x}(h)=\left[a_{h}, f^{-1}\left(a_{h}^{-1} x\right)\right]$, and the morphism $V \longrightarrow Z, x \mapsto s_{x}$, is a two-sided rational inverse to $\phi$.

\section{BirATIONAL MORPHisMS AS BLOWUPS}

Our proof of Theorem 1 in the next section will rely on the following equivariant analogue of [Ha, Theorem 7.17].

Proposition 3. Let $f: X^{\prime} \longrightarrow X$ be a birational proper morphism of $G$-varieties, where $X$ is smooth and $X^{\prime}$ is quasiprojective. Then there exists a $G$-invariant sheaf of ideals $\mathcal{I}$ on $X$ such that $X^{\prime}$ is the blowup of $\mathcal{I}$.

Proof. Let $\sigma: G \times X \longrightarrow X$ be the given action of $G$ on $X$ and $\operatorname{pr}_{2}: G \times X \longrightarrow X$ the projection onto the second factor.

By a theorem of Kambayashi [Ka], there exists an action of $G$ on the projective space $\mathbb{P}^{n}$ (via a representation $G \longrightarrow \mathrm{PGL}_{n+1}$ ) and a $G$-equivariant embedding $X^{\prime} \hookrightarrow \mathbb{P}^{n}$; this yields a $G$-equivariant embedding $i: X^{\prime} \hookrightarrow \mathbb{P}^{n} \times X$.

Here $\mathbb{P}^{n} \times X$ is a projective space over $X$; set $\mathcal{L}=i^{*} \mathcal{O}_{\mathbb{P}^{n} \times X}(1)$ and $\mathcal{T}=$ $\bigoplus_{d=0}^{\infty} f_{*}\left(\mathcal{L}^{d}\right)$, where $\mathcal{L}^{0}=\mathcal{O}_{\mathbb{P}^{n} \times X}$. Let $\mathcal{T}_{1}=f_{*} \mathcal{L}$ be the component of $\mathcal{T}$ of degree one. The action of $G$ on $\mathbb{P}^{n} \times X$ yields a $G$-linearization of the sheaf $\mathcal{T}_{1}$, i.e., an isomorphism $\sigma^{*} \mathcal{T}_{1} \simeq \operatorname{pr}_{2}^{*} \mathcal{T}_{1}$ which satisfies the same cocycle condition as in the definition of $G$-linearization of an invertible sheaf (see, e.g., [MFK, Definition 1.6]); informally speaking, $G$ acts on the pair $\left(X, \mathcal{T}_{1}\right)$.

We refer to the proof of $[\mathrm{Ha}$, Theorem 7.17] for the following facts:

(1) After replacing the embedding $i$ by its $e$-fold embedding for some positive integer $e$ (thus replacing $\mathcal{L}$ by $\mathcal{L}^{e}$ ), we may assume that the graded $\mathcal{O}_{X^{-}}$ algebra $\mathcal{T}$ is generated by $\mathcal{T}_{1}$.

(2) $X^{\prime} \simeq \operatorname{Proj} \mathcal{T}$.

(3) Assume $\mathcal{T}$ is generated by $\mathcal{T}_{1}$ as in (1). If there are an invertible sheaf $\mathcal{M}$ on $X$ and a sheaf of ideals $\mathcal{I}$ on $X$ such that $\mathcal{I} \simeq \mathcal{T}_{1} \otimes \mathcal{M}$, then $X^{\prime}$ is isomorphic to the blowup of $\mathcal{I}$.

The variety $X$ is smooth, and hence, for any sheaf of ideals $\mathcal{F}$ on $X$ of rank one without torsion, its dual $\mathcal{F}^{*}=\mathcal{H o m}\left(\mathcal{F}, \mathcal{O}_{X}\right)$ is an invertible sheaf. (To see this, note that locally at any point $x \in X$, the generator of $\mathcal{F}_{x}^{*}$ is given by the homomorphism $\mathcal{F}_{x} \longrightarrow \mathcal{O}_{X, x}$ which maps the generators of $\mathcal{F}_{x}$, as an $\mathcal{O}_{X, x}$-module of rank one, into elements of $\mathcal{O}_{X, x}$ not having a nontrivial common multiple; such a homomorphism is unique up to an invertible multiple, since the local ring $\mathcal{O}_{X, x}$ is regular, and hence, factorial.)

Thus the second dual $\mathcal{T}_{1}^{* *}$ is an invertible sheaf, and we have an embedding $\mathcal{T}_{1} \hookrightarrow \mathcal{T}_{1}^{* *}$. The $G$-linearization of $\mathcal{T}_{1}$ yields a $G$-linearization of $\mathcal{T}_{1}^{* *}$, and the above embedding is, in fact, an embedding of $G$-linearized sheaves. Taking $\mathcal{M}=\left(\mathcal{T}_{1}^{* *}\right)^{-1}$, we see that $X^{\prime}$ is isomorphic to the blowup of the sheaf of ideals $\mathcal{I}=\mathcal{T}_{1} \otimes\left(\mathcal{I}_{1}^{* *}\right)^{-1}$.

The $G$-linearizations of $\mathcal{T}_{1}$ and $\mathcal{T}_{1}^{* *}$ yield a $G$-linearization of $\mathcal{I}$ and a $G$-linearized embedding $\mathcal{I}=\mathcal{T}_{1} \otimes\left(\mathcal{T}_{1}^{* *}\right)^{-1} \hookrightarrow \mathcal{T}_{1}^{* *} \otimes\left(\mathcal{T}_{1}^{* *}\right)^{-1}=\mathcal{O}_{X}$. This shows that $\mathcal{I}$ is a $G$-invariant sheaf of ideals on $X$. 


\section{Proof of Theorem 1}

First of all, we may assume without loss of generality that $X$ is smooth. Indeed, let

$$
X_{l} \stackrel{\pi_{l}}{\longrightarrow} \ldots \stackrel{\pi_{1}}{\longrightarrow} X_{0}=X
$$

be the canonical resolution of singularities of $X$, as in [V], Theorem 7.6.1] or [BM] Theorem 13.2]. Here $X_{l}$ is smooth, the centers $C_{i} \subset X_{i}$ are smooth and $G$-invariant, and the action of $G$ lifts to the entire resolution sequence (11). Replacing $X$ by $X_{l}$, we may assume that $X$ is smooth.

Now let $\Gamma$ be the closure of the graph of $f: X \rightarrow Y$ in $X \times Y$. By Proposition 2 there exists a proper birational morphism $\phi: Z \longrightarrow \Gamma$ of $G$-varieties, where $Z$ is quasi-projective. Thus we have the following diagram of $G$-equivariant maps:

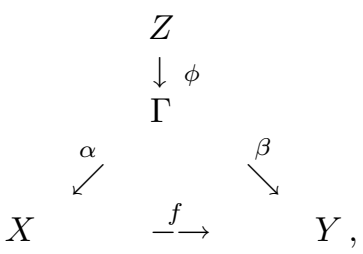

where the maps $\alpha: \Gamma \longrightarrow X$ and $\beta: \Gamma \longrightarrow Y$ are the natural projections to the first and the second factors. Since $Y$ is complete, $\alpha$ is a proper birational morphism, and consequently so is $\alpha \phi: Z \longrightarrow X$. Proposition 3 now says that $\alpha \phi$ is the blowup of a $G$-invariant ideal sheaf $\mathcal{I}$ on $X$. By a theorem of Hironaka [Hi, Main Theorem II] there is a sequence

$$
\pi: X_{m} \stackrel{\pi_{n}}{\longrightarrow} X_{n-1} \cdots \stackrel{\pi_{2}}{\longrightarrow} X_{1} \stackrel{\pi_{1}}{\longrightarrow} X_{0}=X
$$

of blowups with smooth centers such that $\pi^{*} \mathcal{I}$ is locally principal. Moreover, by a theorem of Bierstone and Milman [BM, Theorem 1.10] the sequence $\pi$ can be chosen in a canonical way; in particular, the center of each $\pi_{i}$ can be chosen to be smooth and $G$-invariant; see [BM] Remark 1.5]. Now by the universal property of blowing up (see $\mathrm{Ha}$ Proposition II.7.14]), the composition $(\alpha \phi)^{-1} \pi: X_{m} \rightarrow Z$ is a regular map. Hence, $f \pi=\beta \phi(\alpha \phi)^{-1} \pi: X_{m} \rightarrow Y$ is also regular, as claimed.

\section{ACKNOWLEDGEMENTS}

Some of the work on this paper was done while the first author was participating in the program on Algebraic Groups, Invariant Theory and Applications at the Erwin Schrödinger Institute in Vienna, Austria in the Fall of 2000. He would like to thank the organizers of this program and the Institute staff for their hospitality and support. The second author warmly thanks the Institute of Mathematics of Hebrew University for its hospitality during 1999/2000.

We are grateful to the referee for showing us how to simplify our original arguments.

\section{REFERENCES}

[BM] E. Bierstone, P. D. Milman, Canonical desingularization in characteristic zero by blowing up the maximum strata of a local invariant, Invent. math. 128 (1997), no. 2, 207-302. MR 98e:14010

[Ha] R. Hartshorne. Algebraic geometry. Springer, 1977. MR 57:3116

[Hi] H. Hironaka, Resolution of singularities of an algebraic variety over a field of characteristic 0, I and II, Annals of Math. 79 (1964), 109-326. MR 33:7333 
[Ka] T. Kambayashi, Projective representations of algebraic groups of transformations, Amer. J. Math. 88 (1966), 199-205. MR 34:5826

[MFK] D. Mumford, J. Fogarty and F. Kirwan. Geometric invariant theory. Third enlarged edition, Springer, 1994. MR 95m:14012

[PV] V. L. Popov, E. B. Vinberg, Invariant Theory, in Encyclopedia of Math. Sciences 55, Algebraic Geometry IV, edited by A. N. Parshin and I. R. Shafarevich, Springer-Verlag, 1994. MR 95g:14002

[RY1] Z. Reichstein, B. Youssin, Essential dimensions of algebraic groups and a resolution theorem for G-varieties, with an appendix by J. Kollár and E. Szabó, Canadian J. Math., 52, no. 5 (2000), 1018-1056. MR 2001k:14088

[RY2] Z. Reichstein, B. Youssin, A birational invariant for algebraic group actions, Pacific J. Math., to appear. Available at http://www.math.ubc.ca/ reichst/pub.html

[Su] H. Sumihiro, Equivariant completion, J. Math. Kyoto Univ., 14, no. 1 (1974), 1-28 MR 49:2732

[V] O. E. Villamayor U., Patching local uniformizations, Ann. Scient. Éc. Norm. Sup., 4e série, 25 (1992), 629-677. MR 93m:14012

Department of Mathematics, University of British Columbia, Vancouver, British Columbia, Canada V6T 1Z2

E-mail address: reichst@math.ubc.ca

Department of Mathematics and Computer Science, University of the Negev, Be'er Sheva', IsRael

Current address: Hashofar 26/3, Ma'ale Adumim, Israel

E-mail address: youssin@math.bgu.ac.il 